\title{
Constrained optimization algorithms and automatic differentiation for parameter estimation with application to granulocytic models
}

\author{
Bernd Tibken, Eberhard P. Hofer \\ University of Ulm \\ Department of Measurement, Control and Microtechnology, \\ University of Ulm, D-89069 Ulm, Germany. \\ Tel: +497315026334 . Fax: +497315026301. \\ e-mail: bernd.tibken@e-technik.uni-ulm.de
}

\begin{abstract}
In this paper a dynamic model of granulocytopoiesis is presented which shall be used to estimate cell numbers after bone marrow transfusion. The effects of bone marrow transfusion are interpreted as a change of the initial conditions of the model. The unknown initial conditions of the model are computed on the basis of real patient data with the help of a suitably constructed optimization problem. This optimization problem is solved numerically using an SQP method to compute a stationary point. The step size is controlled via the Armijo rule and the necessary derivatives of the objective function are computed with the automatic differentiation technique.
\end{abstract}

\section{Keywords}

Biomathematical modeling, parameter estimation, real patient data, simulation, optimization, armijo rule, automatic differentiation, system theory

\section{INTRODUCTION}

During recent years biomathematical models to describe granulocytopoiesis have been published (Fliedner and Steinbach, 1987, Hofer, Tibken and Fliedner, 1991, Fliedner, Steinbach and Szepesi, 1988). Although the medical research has almost revealed the structure of granulocytopiesis, the application of these models in the medical treatment of individual patients has been investigated only in recent years (Hofer, Fan and Tibken, 1991, Hofer and Tibken, 1994, Hofer, 1995). In this paper, the model originally proposed by Fliedner and Steinbach is used to quantify the individual perturbation to the hemopoietic 
system due to bone marrow transfusion. This quantification is possible via the estimation of cell numbers for the model from granulocyte measurements from the blood. Based on real patient data the initial numbers of surviving and transplanted cells is calculated, respectively.

\section{MODEL OF GRANULOCYTOPOIESIS}

The model of granulocytopoiesis used consists of 26 differential equations which describe the time evolution of the system. It describes 6 cell and 2 hormone compartments. At the origin of the production of granulocytes a pool S of stem cells is assumed followed by the progenitor pool CBM, the precursor pool $\mathrm{P}$, the maturation pool $\mathrm{M}$, the reserve pool $\mathrm{R}$, and the functional pool $\mathrm{F}$ in the blood. The differential equations describing the model are given by

$$
\begin{aligned}
\frac{d S}{d t}= & \lambda_{S}(1-2 \rho) S \\
\frac{d C B M_{1}}{d t}= & 2(1-\rho) \lambda_{S} S+\left(\alpha_{C}-\lambda_{C}\right) C B M_{1} \\
\frac{d C B M_{i}}{d t}= & \lambda_{C} C B M_{i-1}+\left(\alpha_{C}-\lambda_{C}\right) C B M_{i} \\
& i=2,3, \ldots, 10 \\
\frac{d P_{1}}{d t}= & \lambda_{C} C B M_{10}+\left(\alpha_{P}-\lambda_{P}\right) P_{1} \\
\frac{d P_{i}}{d t}= & \lambda_{P} P_{i-1}+\left(\alpha_{P}-\lambda_{P}\right) P_{i} \\
& i=2,3, \ldots, 10 \\
\frac{d M}{d t}= & \lambda_{P} P_{10}-\lambda_{M} M \\
\frac{d R}{d t}= & \lambda_{M} M-\lambda_{R} R \\
\frac{d F}{d t}= & \lambda_{R} R-\lambda_{F} F \\
\frac{d R e g . I}{d t}= & \varphi_{1}-\lambda_{\text {Reg.I Reg.I }}, \\
\frac{d R e g . I I}{d t}= & \varphi_{2}-\lambda_{\text {Reg.II Reg.II. }} .
\end{aligned}
$$

The pools CBM and $P$ are divided up into 10 subcompartments in order to describe the cell transit time variability. The transit rate $\lambda_{S}$ of the stem cells into division is regulated by the content of all cell compartments in the bone marrow. The fraction $\rho$ of cells returns into the stem cell pool after division while the other cells divide further and mature to granulocytes.

The parameters in the differential equations are choosen to reflect the medical knowledge about the cell transit times and cell amplification due to division. 


\section{ESTIMATION OF CELL NUMBERS}

The mathematical model (1) of granulocytopoiesis consists of a system of nonlinear differential equations given in the previous section. The available real patient data after bone marrow transfusion are measurements of the granulocyte concentration in the blood. Thus, only $F(t)$ for non equidistant time instances is available as measurement. Based on this measurements the initial values for the content of the cell compartments are estimated. For the initial values of general systems of nonlinear differential equations with measurements at non equidistant times no general, systematic and effective method is available. A suitable way to circumvent this problem is to use numerical optimization. software to minimize a problem specific performance index. The only measurable datum is the concentration of $F$ cells in the blood. Thus it is reasonable to perform the estimation using the performance index

$J=\sum_{i=1}^{N}\left(F_{i}-F\left(t_{i}\right)\right)^{2}$

where $t_{i}, F_{i}, i=1, \ldots, N$ are the measurement times and the numbers of granulocytes in the blood, respectively.

The function $J$ is to be minimized with respect to the initial values of the system (1). These initial values are parametrized as follows

$$
\begin{aligned}
S(0) & =\theta_{1} S^{*} \\
C B M_{i}(0) & =\theta_{2} C B M_{i}^{*}, i=1, \ldots, 10 \\
P_{i}(0) & =\theta_{3} P_{i}^{*}, \quad i=1, \ldots, 10 \\
M(0) & =\theta_{4} M^{*} \\
R(0) & =\theta_{5} R^{*} \\
F(0) & =\theta_{6} F^{*}
\end{aligned}
$$

where $S^{*}, C B M_{1}^{*}, \ldots, F^{*}$ are the numbers of cells in the respective compartments in the healthy steady state.

Thus the minimization problem

$J_{\min }=\min _{\theta \geq 0} J(\theta)$

has to be solved in order to estimate the initial cell numbers of a patient based on the observed data. This optimization problem is a constrained optimization with the lower bound 0 on all variables. The numerical methods which have been used and are implemented in MATLAB to solve this problem will be given in the next section.

\section{OPTIMIZATION METHOD}

The optimization problem described in the last section has been solved using a special SQP method. The basic idea is to solve a sequence of easier problems whose solution converges 
to the solution of the original problem. We start with an initial estimate $\theta^{(0)}$ for the minimum and will construct a convergent sequence $\theta^{(k)}$ which satisfies $J\left(\theta^{(k)}\right) \geq J\left(\theta^{(k+1)}\right)$ and $\lim _{k \rightarrow \infty} J\left(\theta^{(k)}\right)=J_{m i n}$. We compute this sequence with the help of the auxiliary problem

$$
\min _{\theta \geq 0}\left(J\left(\theta^{(k)}\right)+\left(\frac{\partial J}{\partial \theta}\left(\theta^{(k)}\right)\right)^{T}\left(\theta-\theta^{(k)}\right)+\frac{1}{2}\left(\theta-\theta^{(k)}\right)^{T}\left(\frac{\partial^{2} J}{\partial \theta^{2}}\left(\theta^{(k)}\right)\right)\left(\theta-\theta^{(k)}\right)\right)
$$

which is constructed from the original problem by a Taylor series expansion of the objective function $J$ at the actual estimate $\theta^{(k)}$ up to the quadratic terms. If the hessian $\frac{\partial^{2} J}{\partial \theta^{2}}\left(\theta^{(k)}\right)$ is positive definit this auxiliary problem will be solved and the solution is denoted by $\tilde{\theta}$. The next iterate $\theta^{(k+1)}$ is then sought between $\theta^{(k)}$ and $\tilde{\theta}$ on the line segment connecting these two points in the parameter space if the direction from $\theta^{(k)}$ to $\tilde{\theta}$ is a descent direction for $J$. If either the hessian is not positive definit or the direction is no descent direction the next iterate is sought in the direction of the negative gradient of $J$ at $\theta^{(k)}$ where the search has to be restricted to the components for which the corresponding components of $\theta^{(k)}$ are not on the boundary of the feasible set, e.g., for the components which are strictly positive. The search is carried out using the armijo rule. This results in a convergent sequence which converges (Kosmol, 1989) to a zero of the gradient of $J$. Thus, if the initial estimate is good enough the convergence to the minimum is guaranteed. The optimization method is implemented using MATLAB and the auxiliary quadratic optimization problem is solved using the corresponding routine in the MATLAB Optimization Toolbox.

The computation of gradient and hessian is carried out using the technique of automatic differentiation. This technique has been implemented in MATLAB using $\mathbf{m}$-files and the necessary computations are done after a call to the corresponding $m$-files. A special Runge-Kutta method has been implemented which uses automatic differentiation for the calculation of the derivatives of the numerical solution to (1) with respect to the parameters $\theta_{1}, \ldots \theta_{6}$.

\section{CONCLUSION AND OUTLOOK}

In this paper a special optimization method has been presented which has been implemented using MATLAB. It is based on a quadratic approximation of the function to be minimized and takes simple bounds on the variables into account in each step. Thus, infeasible points are never generated during the optimization. This is important because in the application in mind the variables are cell numbers which have to be positive in order to have an biological interpretation. The convergence near the optimum is quadratic and first results look very promising.

The method has been applied to the estimation of cell numbers after severe irradiation and bone marrow transplantation after leukemia treatment.

Future work will concentrate on the application of Quasi Newton Methods which need only gradient information and no hessian. Thus, eventually the necessary computer time to perform an estimation can be reduced drastically. 


\section{REFERENCES}

Hofer, E.P., Fan, Y. and Tibken, B. (1991) Extraction of Rules for Model Based Estimation of Granulocytopoiesis, in The 5th German-Japanese Seminar on Nonlinear Problems in Dynamical Systems - Theory and Applications -. (ed. M. Frik), Duisburg.

Hofer, E.P., Tibken, B. and Fliedner, T.M. (1991) Modern control theory as a tool to describe the biomathematical model of granulocytopoiesis, in Analyse Dynamischer Systeme in Medizin, Biologie und Ökologie. (eds. D.P.F. Möller, O. Richter), Informatik Fachberichte 275, Springer, Berlin.

Hofer, E.P. and Tibken, B. (1994) A Clinical Decision Support System for the Treatment of Irradiated Persons Based on a Biomathematical Model of Granulocytopoiesis, in AUTOMATIC CONTROL 12th Triennial World Congress of IFAC (eds. G.C. Goodwin, R.J. Evans), Pergamon, 3.

Hofer, E.P. (1995) On the Way to a Decision Support Tool for Treatment of Irradiated Persons Based on Hemopoietic Models. Journal of SICE, 34, 410-7.

Fliedner, T.M., Steinbach, K.-H. and Szepesi, T. (1988) Hematological indicators in the determination of clinical management strategies in radiation accidents, in International Conference on Biological Effects of Large Dose Ionizing and Non-Ionizing Radiation, Hangzhou, China.

Fliedner, T.M. and Steinbach, K.-H. (1987) Simulationsmodelle von Perturbationen des granulozytären Zellerneuerungssystems, in Modelle der Pathologischen Physiologie. (eds. W. Doerr, H. Schipperges), Springer, Berlin.

Kosmol, P. (1989) Methoden zur numerischen Behandlung nichtlinearer Gleichungen und Optimierungsaufgaben. Teubner, Stuttgart. 